\title{
Large retrosternal goiter: an otolaryngological perspective-case series and review of literature
}

\begin{abstract}
Introduction: Retrosternal goiter can cause respiratory distress, dysphagia, compression of great vessels, and even sudden death. Surgery is the only effective treatment. The presence of retrosternal extension is an indication for surgery even in the absence of clinical symptoms as it will continue to grow and cause pressure symptoms eventually. Intra operative and post operative airway management also poses a challenge in such cases. Most retrosternal goitres can be excised via a cervical approach; however, in cases of large size with very inferior extent, abnormal vasculature, in cases of recurrent goitres or thyroid cancer, a midline sternotomy may be necessary for exposure, safety, and completion of the excision.
\end{abstract}

Methods: Five patients of multinodular goiter with retrosternal extension from $8 \mathrm{~cm}$ to $10 \mathrm{~cm}$ have been successfully managed at our centre from Jan 2015 to Jun 2016. We analysed the medical records of these five patients retrospectively along with peri operative management and one nodal case with maximum retrosternal extension of $10 \mathrm{~cm}$, which was removed via transcervical approach without the need for sternotomy is described.

Conclusion: Large retrosternal goiter presents a challenge to the otolaryngologist due to their size and location. Intrathoracic goitres must be removed as soon as possible to reduce the risk of tracheomalacia, invasion to the adjacent structures, and distal organ metastasis due to the possibility of malignancy.

Keywords: retrosternal, goiter, sternotomy, transcervical, surgical approach, cervicothoracic isthmus, thyroid gland, tracheomalcia
Volume 6 Issue I - 2017

Inderdeep Singh, ID Singh, Vikas Gupta, Sheetal Raina, Sunil Goyal, Manoj Kumar Department of Otolaryngology Head Neck Surgery Command Hospital Southern Command, India

Correspondence: ID Singh Department of Otolaryngology Head Neck Surgery Command Hospital Southern Command Pune, India,Tel +91-7767834137, Email dridsingh@hotmail.com

Received: November 04, 2016 | Published: January 17, 2017
Abbreviations: RG, retrosternal goitre; CECT, computed tomography; CTVS, cardio thoracic vascular surgery; FNAC, fine needle aspiration cytology; Tc99, technetium 99; RLN, recurrent laryngeal nerve; HPE, histo pathological examination; IJV, internal jugular vein

\section{Introduction}

Retrosternal goitre (RG) was first described by Albrecht von Haller in 1749 and first operated by Klein in $1820 .{ }^{1-4}$ There is no uniform definition of retrosternal goiter. Extension of the thyroid gland below the thoracic inlet has been also described in literature variously, like substernal, retrosternal, intrathoracic, or mediastinal goiter. However, various authors have defined different criteria to call it as RG. These include a thyroid gland extending $3 \mathrm{~cm}$ below cervicothoracic isthmus below at CT, performed with hyper extended neck $^{5}$ or extension of the gland below the fourth thoracic vertebra. ${ }^{6,7}$ The most commonly accepted definition of RG describes a goitre as substernal or retrosternal when $\geq 50 \%$ total bulk of thyroid tissue resides below thoracic inlet. ${ }^{8,9}$

Management of RG, depending upon their size and extension can be a daunting task for surgeon, where the surgical team has to be in coherence with multidisciplinary team including anaesthetist and cardiothoracic surgeon who has to be on standby in case of difficulty in extrusion of the tumour. Most of RG can be resected safely through a cervical incision. ${ }^{8}$ In minority of cases $1-11 \%$, a sternotomy is necessary to have complete exposure of the goitre and remove mediastinal compression. ${ }^{4}$ Also, combined cervical with sternotomy approach can lead to more intra-operative complications and slower post-operative rehabilitation.

\section{Clinical data}

\section{Patient information}

We treated 05 female patients, in the age group of 38-60years (median age of 51). Mean duration of disease among patients was 6-36months. The chief complaint of all patients was anterior neck mass, without any compressive or pressure symptoms, except one patient who had breathlessness on exertion. On examination, all patients had firm, non tender palpable anterior neck mass which moved on deglutition, with multiple nodules could be palpated involving both lobes. The inferior border of the mass was not palpable. Vocal cords were mobile $\mathrm{B} / \mathrm{l}$ on Hopkins telescopy. There were no signs of compression/pressure due to retrosternal extension. Our patients with lesion had minimum dimension of $4 \mathrm{~cm} \times 5 \mathrm{~cm} \times 8 \mathrm{~cm}$ to the largest measuring $4 \mathrm{~cm} \times 8 \mathrm{~cm} \times 14 \mathrm{~cm}$ (AP $\times$ TR $\times$ CC) with retrosternal extension ranging from $8 \mathrm{~cm}$ to largest of $10 \mathrm{~cm}$ (Figure 1).

\section{Workup}

All patients underwent thyroid function test, US guided FNAC, USG neck, CECT scan, Thyroid scan. On FNAC, all patients were reported as benign nodular goiter. All patients were biochemically and clinically euthyroid.

Imaging in form of radiographs of the neck and chest revealed a soft tissue density lesion in the superior mediastinum with welldefined lateral margins; however the inferior margin was not well defined. The trachea was central with no evidence of any compression (Figure 2A \& 2B).

USG neck and CECT scan in view of retrosternal extension, revealed minimum extension of $8 \mathrm{~cm}$ to maximum of $10 \mathrm{~cm}$. All retrosternal extension were descending to the anterior mediastinum, 
just abutting the level of aortic arch. The mass was causing mild tracheal luminal compromise in 03 cases and in the postero inferior aspect, the lesion was indenting and slightly displacing the aortic arch in 02 cases; however, the intervening fat planes were maintained. Laterally, the lesion was causing indentation of bilateral Internal Jugular Vein in all cases, 03 on right and 02 on left. Rest of the great vessels were normally visualised (Figure 3A, 3B \& 3C).

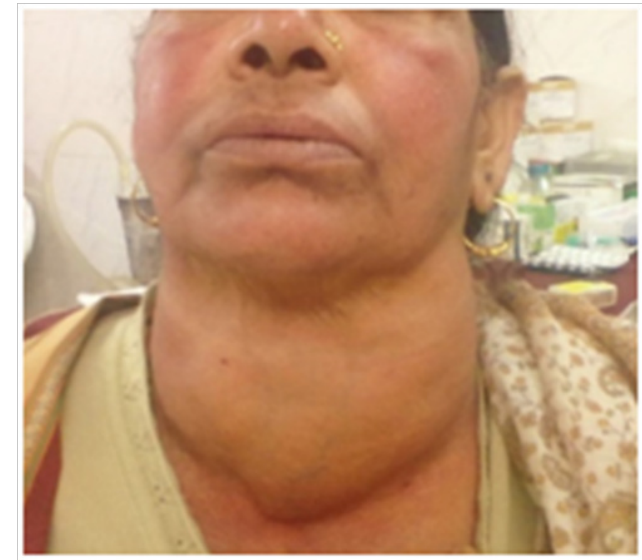

Figure I Gross enlargement of $\mathrm{B} / \mathrm{l}$ thyroid lobes with indistinct inferior border.

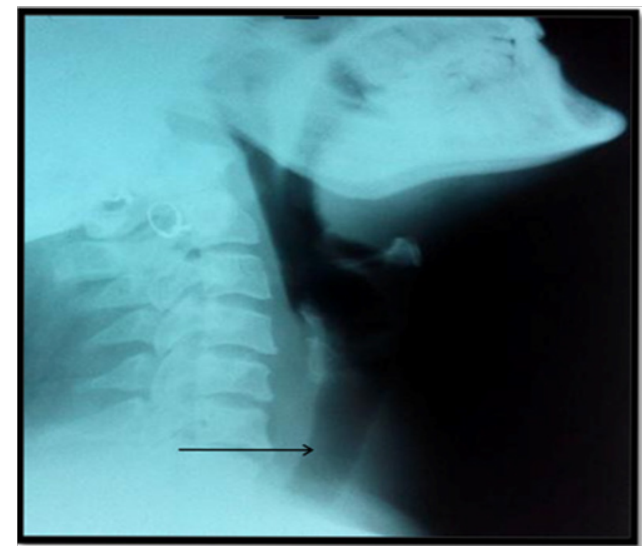

Figure 2A Soft tissue swelling (likely thyroid gland) in anterior aspect of lower cervical region with no trachea compression (arrow).

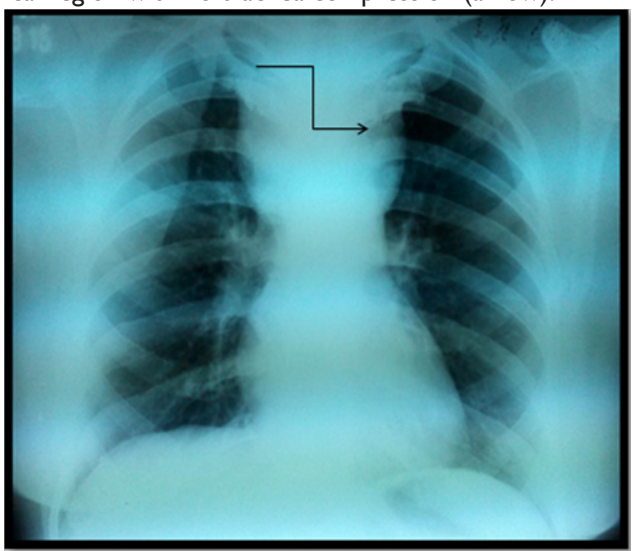

Figure 2B Soft tissue swelling in superior mediastinum with well-defined lateral margins (arrow). Inferior margin is ill defined.

$\mathrm{Tc}^{99 \mathrm{~m}}$ thyroid scan was done which was suggestive of grossly enlarged thyroid gland with retrosternal extension. The uptake was heterogeneous with multiple interspersed cold regions, the overall uptake ranging from $2-8 \%$ (Figure 4 ).

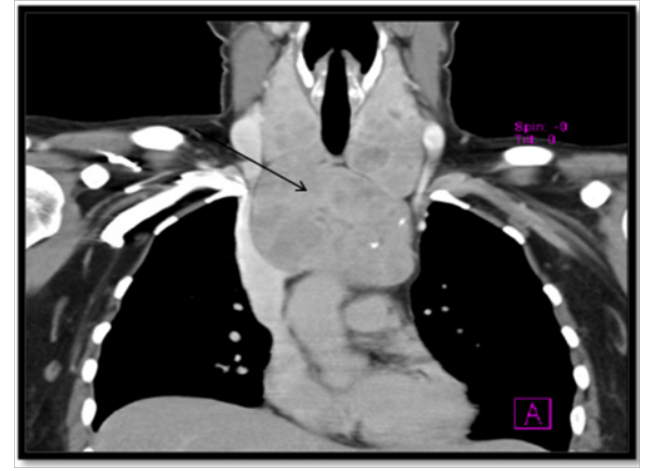

Figure 3A CT image: Coronal section showing retrosternal extension of goiter (arrow).

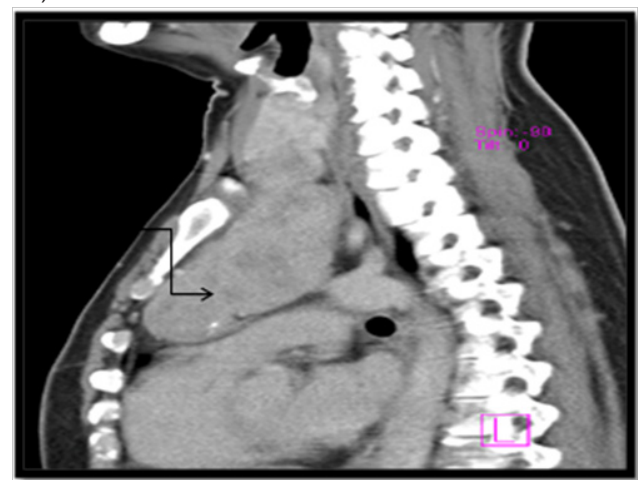

Figure 3B CT image: Sagittal section showing retrosternal extension, approx. $10 \mathrm{~cm}$ from superior most aspect of manubrium (arrow).

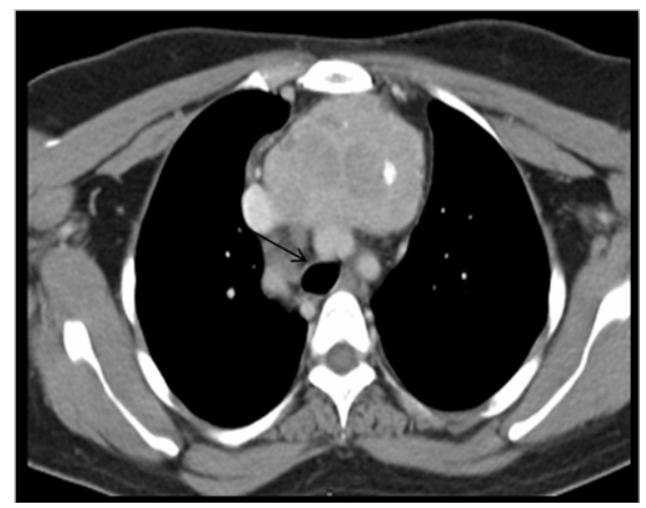

Figure 3C CT image:Axial section at the level of T2-T3 showing retrosternal extension of goitre upto the aortic arch (arrow).

\section{Treatment}

After thorough workup, cases were discussed in multidisciplinary clinics with cardio-thoracic surgeons, in case need arise for sternotomy, and anaesthesiologist in view of anticipated airway complications arising intra operative or post operative period. Patients were counselled adequately and informed consent was sought for possible RLN injury, post op tracheostomy, hypocalcemia, with specific consent for possible sternotomy. Rigid bronchoscope with ventilator connector was kept on standby in anticipation of difficult intubation. Trachea reinforcing (flexo-metallic) endotracheal tube was inserted in all cases so that even the manipulation pressure would not cause any significant compression of the tracheal tube. The left radial artery was canulated to monitor airway pressure to ensure a safe surgery. A continuous intra-arterial pressure monitoring was done to 
see the compression of the great vessels if any during the course of the surgery in all cases. Preparation for possible sternotomy was also done. Prophylactic antibiotic was administered prior to incision as per existing guidelines and institutional protocols.

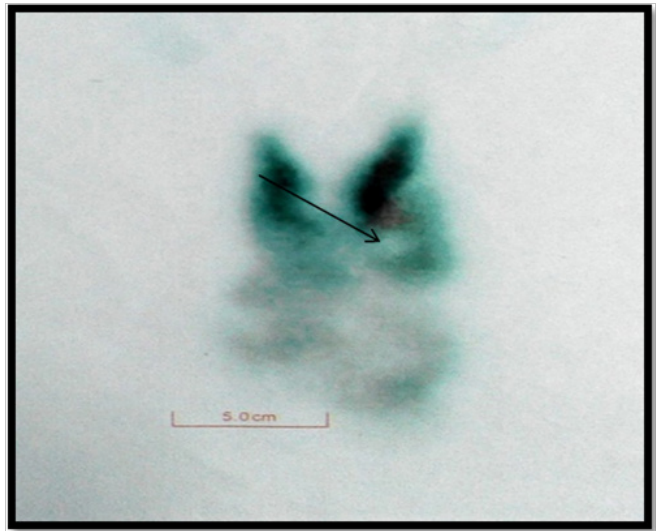

Figure $4 \mathrm{Tc} 99 \mathrm{~m}$ pertechnate images; grossly enlarged thyroid gland with retrosternal extension. Heterogenous uptake with multiple interspersed cold regions. Overall uptake: $8 \%$ (arrow).

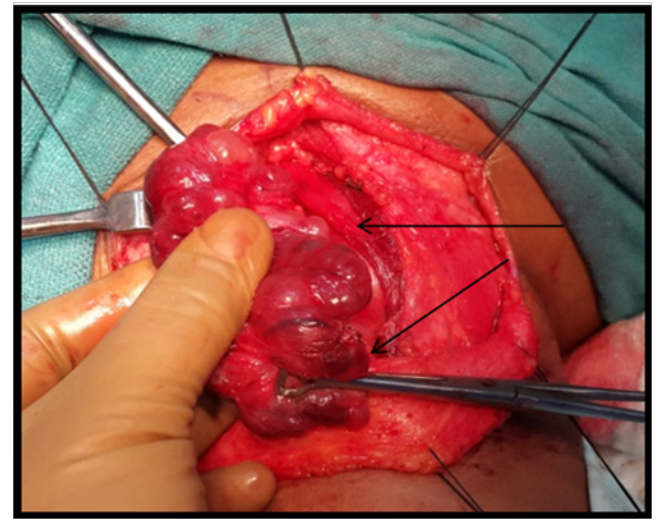

Figure 5A Multiple nodules on the surface of thyroid gland (arrow). Both the lobes dissected out after preserving parathyroid's and RLN.

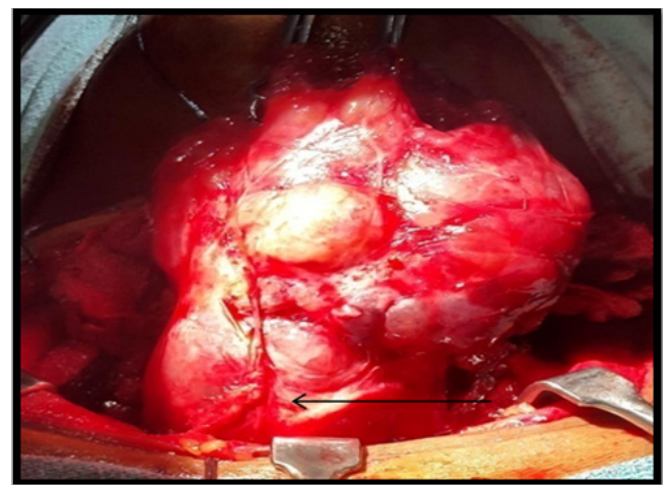

Figure 5B Retrosternal extension of the goitre visualized (arrow).

All patients underwent total thyroidectomy. Hereafter, the surgical technique employed and introp findings for the case with maximum retrosternal goiter (upto $10 \mathrm{~cm}$ ) are described. A low lying horizontal neck crease incision about $1-1.5 \mathrm{~cm}$ above sternal notch was taken in a fully extended neck and the strap muscles retracted after the midline split. The dissection started from the superior pole from the left side being comparatively smaller to right side which was occupying the major part of retrosternal and making the inferior most part. This was done to ensure safety of atleast one RLN. Superior thyroid artery was identified and its branches ligated individually after identifying and preserving EBSLN. In view of large dimensions of the thyroid gland it was judged prudent to identify RLN via superior dissection after identifying the Cricothyroid (CT) joint. The superior parathyroids identified approximately $1 \mathrm{~cm}$ caudally to CT joint and dorsal to RLN on both the sides and preserved. The inferior thyroid artery branches were ligated on the left side after preserving left parathyroid ventral to RLN but on the right side inferior thyroid artery was ligated in its main trunk. On Right, inferior parathyroid gland could not be identified. The left lobe along with its retrosternal extension was removed first. The right lobe which had the main retrosternal extension was dissected completely from the lateral side and then was cut into two halves after identifying the right RLN and the carotid sheath as to give more space for the manipulation of main retrosternal part. Blunt but gentle manipulation of the retrosternal extension in the superior mediastinum was done with the fingers above the plane of the trachea and the carotid artery. The assistant surgeon applied the traction superiorly and releasing it intermittently to avoid a shearing effect or excessive pressure on the great vessels. The manipulations were avoided lateral and below the carotid vessels or the trachea, to avoid devascularisation of trachea. The tracheo-esophageal groove was not violated. In the intrathoracic part all the thick bands or adhesions were palpated for any pulsation with the two fingers and they were dissected into thinner bands before their removal as to avoid any inadvertent trauma to any vessels. The lower pole was palpable intraoperatively and it was gently manipulated by the index finger to free any adhesions and the lobe was pulled up. A thickened thyroid ima artery was visualised and at the lower pole of the retrosternal extension was ligated and the entire retrosternal part delivered. Haemostasis was achieved and a surgical drain was placed and wound closed in layers. The entire thyroid gland was sent for HPE.

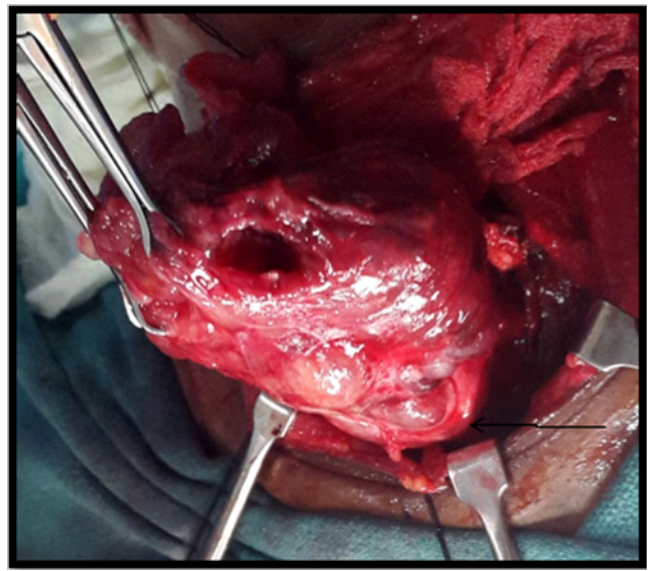

Figure 5C Retrosternal extension of the goitre with the lower pole at the level of thoracic inlet (arrow); being removed after gentle manipulation.

All patients remained intubated electively in the post-operative period for observation in anticipation of tracheomalacia and were extubated on post-operative day one. A tracheostomy set was kept ready at the time of extubation in case of any airway emergency. In the post-operative period, 03 patients developed transient hypocalcaemia on post-op day 2 which lasted for a day and was managed effectively with IV Calcium Gluconate and oral calcium supplements. The patient was discharged after drain removal by post-op day5. In post op period, $\mathrm{b} / \mathrm{l}$ vocal cord movements were assessed by fibre-optic laryngoscope, the cords were mobile in all cases, except in one case where there was Right vocal cord paresis, which recovered within six weeks. Tracheomalcia was not identified in any case. HPE was consistent 
with colloid goiter in all cases. All patients are on regular follow up with levothyroxine supplements and 01 patient are on calcium supplements (Table 1).

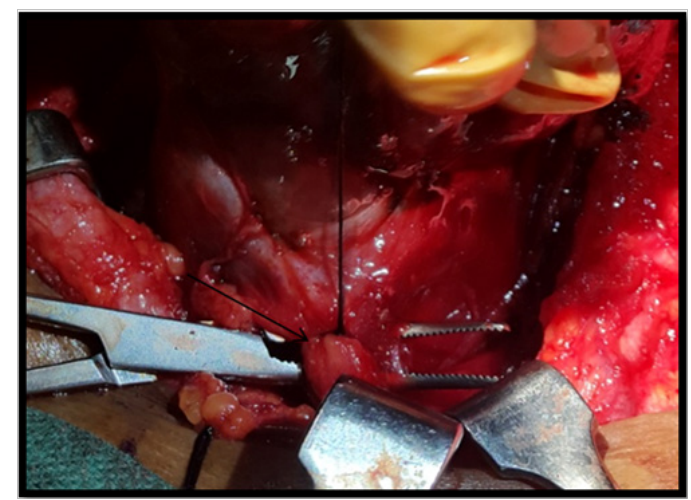

Figure 5D Ligating the thick thyroid ima artery. Note the engorged and thickened artery (arrow).

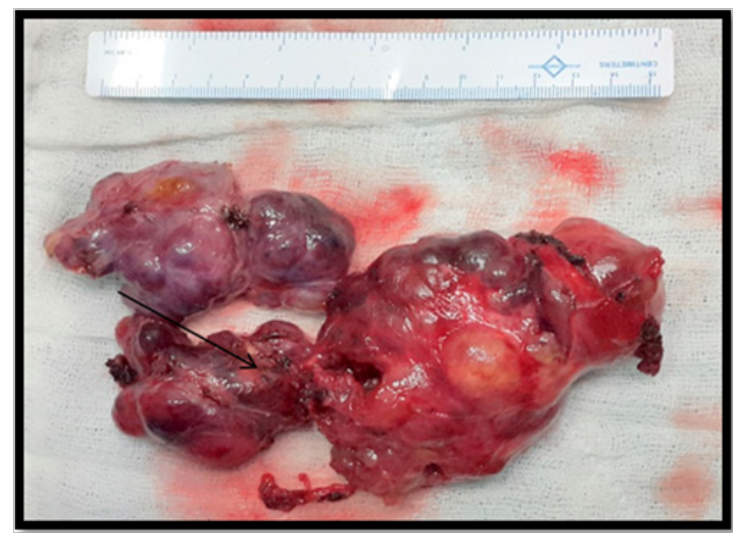

Figure 5E Ligating the thick thyroid ima artery. Note the engorged and thickened artery (arrow).

Table I Salient features of patients

\begin{tabular}{|c|c|c|c|c|c|c|c|}
\hline $\begin{array}{l}\text { S. } \\
\text { No }\end{array}$ & Age & Gender & $\begin{array}{l}\text { Duration of } \\
\text { Symptoms }\end{array}$ & $\begin{array}{l}\text { Size of Retrosternal } \\
\text { Extension on CECT }\end{array}$ & $\begin{array}{l}\text { Sternotomy } \\
\text { Employed (Y/N) }\end{array}$ & $\begin{array}{l}\text { Post-Operative } \\
\text { Complications }\end{array}$ & HPE Report \\
\hline I & $38 y$ & Female & 6 months & $8 \mathrm{~cm}$ & No & Transient hypocalcemia & Colloid goiter \\
\hline 2 & $40 y$ & Female & 36 months & $10 \mathrm{~cm}$ & No & Transient vocal cord paresis $\mathrm{Rt}$ & Colloid goiter \\
\hline 3 & $48 y$ & Female & 18 months & $9 \mathrm{~cm}$ & No & Nil & Colloid goiter \\
\hline 4 & $51 y$ & Female & 24 months & $8.5 \mathrm{~cm}$ & No & Nil & Colloid goiter \\
\hline 5 & $60 y$ & Female & 24 months & $9.5 \mathrm{~cm}$ & No & Hypocalcemia & Colloid goiter \\
\hline
\end{tabular}

\section{Discussion}

RG occurs when the thyroid enlarges downwards into the chest. Majority of RG are extensions from the neck, however intrathoracic goitres has also been reported. The definition of RG has been a matter of debate and it varies from author to author.

Katlic et al., ${ }^{9}$ suggested that RG was when more than $50 \%$ of goitre is below plane of thoracic inlet. Candela et al., ${ }^{10}$ defined as any goitre that descended below the plane of thoracic inlet or going into anterior mediastinum for more than $2 \mathrm{cms} .{ }^{10}$ Goldenburg and co workers as early as 1957 defined RG as one reaching the level of fourth thoracic vertebra. ${ }^{11}$ Most widely accepted classification of RG and management depending on its extension into mediastinum has been proposed by Huins et al., ${ }^{1}$ They defined three grades of goitre namely, grade I to the level of the aortic arch, grade II to the level of the pericardium and grade III below the level of the right atrium.

RG has been reported in $1-20 \%$ of all patients undergoing thyroidectomy. ${ }^{12,13} \mathrm{RG}$ is most frequently found in the fifth or sixth decade of life, with a female/male ratio of $4: 1{ }^{14}$ The vast majority of RG $(85-90 \%)$ are located in the anterior mediastinum. Posterior mediastinal goiters are uncommon comprising $10-15 \%$ of all mediastinal goiters. ${ }^{15}$

RG show, in most cases, a slow growing enlargement, which usually remain asymptomatic for many years; about $20-40 \%$ of retrosternal goitres are symptomatic ${ }^{14}$. They carry a risk of malignancy similar to the cervical goiters ${ }^{4}$. The most common symptoms are related to compression of the airway, esophagus, RLN and represented by dyspnoea, choking, inability to lie straight, dysphagia and hoarseness 2. Superior vena cava syndrome due to SVC obstruction and Horner's syndrome due to compression of sympathetic chain is less common.
Anterior mediastinum is most common site for retrosternal goitre extension. It displaces trachea causing its compression. The majority of patients, in addition to neck swelling may present with shortness of breath or stridor. Other symptoms include hoarseness, dysphagia, and about $50 \%$ of patients may be asymptomatic. ${ }^{16}$ Thyrotoxicosis symptoms were reported in less than $10 \%$ of case. ${ }^{17}$

In the diagnostic management of RG, CECT Thorax is the gold standard radiological investigation and preoperative CT scan should be done routinely for suspicious retrosternal goiter. ${ }^{5}$ The relationship of RG, with trachea, esophagus, great vessels is well appreciated and helps in planning the surgical approach, which may require inputs from other surgical team and also provides information to anaesthesiologist about the airway. Contrast enhanced MRI can also be performed in conjunction with CECT, which may add information regarding surrounding tissue planes to the thyroid mass. Casella et al. ${ }^{15}$ found that extension of the goitre below the level of the aortic arch appeared to be a significant predictive factor for the need for sternotomy. Conversely, the lack of radiologic extension beyond the aortic arch predicted successful trans cervical removal of mediastinal goiters without sternotomy. ${ }^{15}$

Surgery is the definite treatment of RG, the earlier RG is tackled, more safer it is for patient, in terms of pre op, intra op and post op morbidity.

Majority of RG can be delivered and resected safely through standard cervical approach, however, this approach has inherent risks of damage to great vessel during blind manipulation and poor control of haemorrhage thereafter due close space and therefore this approach is generally avoided in RG with extension beyond aortic arch. In our experience the retrosternal goitres upto the size of $10 \mathrm{~cm}$ can be removed via cervical route with careful digital manipulations. 
De Perrot et al. ${ }^{5}$ has also highlighted the need of sternotomy in goitres larger than $10 \mathrm{~cm}$, in case of malignancy, involvement of posterior mediastinum and or ectopic goiter, ${ }^{5}$ which has also been identified by Cohen et al., ${ }^{6}$

Preservation of parathyroids, RLN, EBSLN, as in regular thyroidectomy cannot be emphasised more. RLN should localised preferably early and by superior approach or lateral approach, as it is not uncommon for the nerve to be riding over a hyperplastic nodule, making it vulnerable.

The role of assistant surgeon is important while manipulation as he gives the necessary traction with intermittent release so that the pressure is just adequate for the surgeon to continue digital dissection. The assistant surgeon also helps in pulling out the retrosternal part by his digital pressure from the opposite side when the surgeon manipulates from the lateral and the anterior sides or while palpating the planes for any pulsating vessels. During the final delivery one has to keep in mind by palpating the fibrous attachments of any aberrant blood vessels like high riding innominate artery which may be asymmetry in its position and thyroid ima artery which may be thick and can cause troublesome bleeding requiring sternotomy exploration. Sometimes there can be difficulty in delivering the retrosternal segment due to narrow retrosternal space. In such situation Charles Proye described that the normal or the smaller lobe should be excised first to provide more space in the neck, similar technique was employed in our case series. Charles Proye also described Toboggan technique, which is done by placing heavy silk into the cervical component to provide traction and then placing more sutures in series in order to bring the retrosternal extension into view. ${ }^{18}$ However this technique has limited application in presence of soft colloid goiter. ${ }^{19}$

The most important predictive factor as to whether goitre can safely be removed through a cervical approach is the presence of a clear tissue plane around the nodule in the mediastinum on preoperative imaging. If such a clear plane is not present, preparations should be made for sternotomy. ${ }^{13} \mathrm{Ahmed}$ et al., ${ }^{12}$ used extension beyond the aortic knuckle on chest X-ray as their landmark for the depth of substernal extension. ${ }^{12}$

Mussi et al., ${ }^{20}$ believed that sternotomy should be employed when a goitre could not be extracted from the chest with "gentle manoeuvres," as well in cases of all recurrent and aberrant goiters. ${ }^{20}$ Sand et al., ${ }^{21}$ employed sternotomy when excessive traction is required during surgery, when the most inferior extent of the nodule cannot be palpated, in cases of revision surgery, in the setting of acute tracheal compression, severe venous obstruction, malignancy, and uncertain preoperative diagnosis. ${ }^{21}$ Sancho felt that nodules that extended inferiorly to the level of the carina placed patients at high risk for sternotomy. ${ }^{22}$ Randolph recommended sternotomy for malignant sub sternal nodules, posterior mediastinal goitre with contra lateral extension, mediastinal goitres with mediastinal blood supply, goitres causing superior vena cava syndrome, revision cases, in the setting of difficult delivery from the chest, significant haemorrhage, and when the diameter of the mediastinal nodule significantly exceeds the diameter of the thoracic inlet. ${ }^{23}$

Sternotomy approach is also associated with complications and post op morbidities, like trauma to mediastinal structures, pmeumothorax, mediastinitis, sternal dehiscence and osteomyelitis. In cases of long standing large goiters, the trachea could lose its structural strength and can lead to tracheomalacia. Therefore, tracheomalcia should be anticipated in post op period, and it is advisable that patient should remain intubated for atleast a day and during extubation tracheostomy set should be kept ready. ${ }^{24}$
In our case series of five patients, the recurrent laryngeal nerves both sides could be identified in all cases and the both superior parathyroid glands were preserved in all cases; however, postoperative complications in the form of transient hypoparathyroidism occurred in three cases which resolved over a period of one week and transient vocal cord paresis right side occurred in two cases which gradually resolved over a period of a four weeks. All the five cases postoperative were kept overnight on endotracheal tube intubation and after extubation on the same day they all underwent fibreoptic laryngoscopy to assess the vocal cord mobility and tracheal airway examination. None of our cases required postop tracheostomy or prolonged intubation and none of our cases showed any signs of tracheomalacia. There were no post-operative respiratory complications or any post-operative bleeding and neither was there any permanent RLN palsy.

\section{Conclusion}

Large retrosternal goitre presents a challenge to otolaryngologist, whether to go for cervical or sternotomy approach. Cervical approach provides benefits in term of patient morbidity but not without its set of possible complications described earlier. In our case series we preferred cervical approach though it is challenging. Cervical approach is possible with meticulous finger dissection, good assistance without much morbidity to patient, hence it is recommended to start with cervical approach with a standby thoracic team ready for thoracotomy if need arises.

\section{Conflicts of interest}

Informed consent taken from all the patients to publish the photographs and medical data for the purpose of publication in medical science.

\section{Acknowledgments}

None.

\section{Funding}

None.

\section{References}

1. Huins CT, Georgalas C, Mehrzad H, et al. A new classification system for retrosternal based on a systematic review of its complications and management. Int J Surg. 2008; 6(1):71-76.

2. Erbil Y, Bozbora A, Barbaros U, et al. Surgical management of substernal goiters: clinical experience of 170 cases. Surg Today. 2004;34(9):732-736.

3. Madjar S, Weissberg D. Retrosternal goiter. Chest. 1995;108(1):78-82.

4. White ML, Doherty GM, Gauger PG. Evidence-based surgical management of substernal goiter. World J Surg. 2008;32(7):1285-1300.

5. De Perrot M, Fadel E, Mercier O, et al. Surgical management of Mediastinal goiters: when is a sternotomy required? J Thorac Cardiovasc Surg. 2007;55(1):39-43.

6. Cannon CR, Lee R, Didlake R. Management of the substernal goiter: a team approach. J Miss State Med Assoc. 2010;51(7):179-182.

7. Flati G, De Giacomo T, Porowska B, et al. Surgical management of substernal goiters: when is sternotomy inevitable? Clin Ter 156(5): 191195.

8. Desouza, Smith PE (1983) Reterosternal goitre. J Otolaryngol. 2005;12(6):393396 
9. Katlic MR, Grillo HC, Wang CA. Substernal goiter. Analysis of 80 patients from Massachusetts General Hospital. Am J Surg. 1985;149(2):283-287.

10. Candela G, Varriale S, Di Libero L, et al. Surgical therapy of goiter plunged in the mediastinum. Considerations regarding our experience with 165 patients. Chir Ital. 2007;59(6):843-851.

11. Goldenburg IS, Lindskog GE. Differential diagnosis, pathology and treatment of substernal goiter. J Am Med Assoc. 1957;163(7):527-529.

12. Ahmed ME, Ahmed EO, Mahadi SI. Retrosternal goiter: the need for median sternotomy. World J Surg. 2006;30(11):1945-1948.

13. Batori M, Chatelou E, Straniero A. Surgical treatment of retrosternal goiter. Eur Rev Med Pharmacol Sci. 2007;11(4):265-268.

14. Cohen JP. Substernal goiters and sternotomy. Laryngoscope. 2009;119(4):683-688.

15. Rugiu MG, Piemonte M. Surgical approach to retrosternal goitre: do we still need sternotomy? Acta Otorhinolaryngol Ital. 2009;29(6):331-338.

16. Casella C, Pata G, Cappelli C, et al. Preoperative predictors of sternotomy need in mediastinal goiter management. Head Neck. 2010;32(9):1131-1135.

17. Moron JG, Singer JA, Sardi A. Retrosternal goitre: A six year institutional review. Am Surg. 1998;64(9):889-893.
18. Hedayati N, McHenry CR. The clinical presentation and operative management of nodular and diffuse substernal thyroid disease. Am Surg. 2002;68(3):245-251.

19. Proye CAG. Substernal goitre. Surgical technique. Curr Pract Surgery. $1993 ; 5: 72-77$

20. Saddler GP, Clarck OH, Heerden JA, et al. Thyroid and parathyroid. In: Shwartz \& Shires (Eds.), Principle of surgery. McGraw-Hill, New York. 1999. p. 1689-1692.

21. Mussi A, Ambrogi MC, Lacconi P, et al. Mediastinal goiters: when the trans thoracic approach? Acta Chir Belg. 2000;100(6):259-263.

22. Sand ME, Laws HL, McElvein RB. Substernal and intrathoracic goiter. Reconsideration of surgical approach. Am Surg. 1983;49(4):196-202.

23. Sancho JJ, Kraimps JL, Sanchez-Blanco JM, et al. Increased mortality and morbidity associated with thyroidectomy for intrathoracic goiters reaching the carina tracheae. Arch Surg. 2006;141(1):82-85.

24. Randolph GW. Surgery of the Thyroid and Parathyroid Glands. (2nd edn), Elsevier Saunders, Philadelphia, USA. 2013. p. 75-76.

25. Barbuscia MA, Gorgone S, Di Pietro N, et al. Substernal goiter: pre, intra and post operative problems. Chir Ital. 2005;57(3):301-307. 